Pacific Journal of Mathematic 


\title{
EXTREMAL STRUCTURE OF STAR-SHAPED SETS
}

\author{
F. E. TIDMORE
}

\begin{abstract}
It is shown that the convex kernel of a compact starshaped subset $S$ of a finite-dimensional linear topological space $L_{n}$ is determined by the $(n-1)$-extreme points of $S$. The cardinality of the set of $k$-extreme points is determined for compact star-shaped sets of dimension greater than two. Also given is the result that any compact star-shaped subset $S$ of $L_{n}$ contains a countable set of $(n-1)$-extreme points which determines the convex kernel of $S$. Another result is that a compact nonconvex star-shaped set $S$ in a locally convex space $L$ is determined by the convex kernel of $S$ and the subset of points that are extreme in $S$ relative to the convex kernel of S.
\end{abstract}

The convex kernel of a star-shaped set $S$ will be denoted by $c k S$, the line segment $\{\alpha x+(1-\alpha) y: \alpha \in[0,1]\}$ will be denoted by $x y$, the ray $\{\beta y+(1-\beta) x: \beta \geqq 1\}$ will be denoted by $x y^{\infty}$ and $L(x, y)$ will denote the line containing $x$ and $y, x \neq y$. The convex hull of a set $S$ will be denoted by conv $S$. The notation intv $S$ will denote the interior of $S$ relative to the minimal flat that contains $S$. The set $\{x: f(x)=\alpha\}$, where $f$ is a linear functional, will be denoted $[f: \alpha]$. Set-theoretic difference will be denoted by $\backslash$, and the closure of a set $S$ will be denoted by cl $S$.

The concept of $k$-extreme point was introduced by Asplund [1].

Definition 1. If $S$ is a subset of a linear space $L$, a point $x \in S$ is a $k$-extreme point of $S$ if no $k$-simplex $\Delta$ exists such that $x \in \operatorname{intv} \Delta \subset S$.

For a subset $S$ of a linear space $L, S_{x}$ will denote the $x$-star of $S$ determined by the point $x \in S$; that is, the set of points $y$ such that $x y \subset S$. If $S$ is a closed (compact) subset of a linear topological space $L$, then for any $x \in S, S_{x}$ is a closed (compact) set. If $T \subset S$, let

$$
S_{T}=\bigcap_{x \in T} S_{x}
$$

A point $p$ belongs to the convex kernel of $S$ if, and only if, $x p \subset S$ for all $x \in S$, which is true if, and only if, $p \in S_{x}$ for all $x \in S$. Thus $c k S=S_{S}$, which motivates the following definition.

Definition 2. In a linear space $L$ a subset $T$ of a star-shaped 
set $S$ is said to star-generate the convex kernel of $S$ if $c k S=S_{T}$. Such a subset $T$ is said to be a star-generating set for $c k S$.

THEOREM 1. Let $S$ be a compact star-shaped subset of $L_{k+1}$. Then the set $S(k)$ of k-extreme points of $S$ is a star-generating set for cks.

Proof. Without loss of generality, suppose that $0 \in c k S$. If $S=$ $c k S$, then $S$ is convex and $S_{x}=S$ for each $x \in S$ and the result follows since $\varnothing \neq S(1) \subset S(k)$. Let $p \in S \backslash c k S$. Then there exists a point $y \in S$ such that $p y \not \subset S$. Since $S$ is compact, $y$ can be chosen such that $S \cap$ intv $p y^{\infty}=\varnothing$. Since $p y \not \subset S$, there exists a point $z \epsilon$ (intv $p y) \backslash S$. If $y \in S(k)$, then $p \notin S_{y}$ implies $p \notin S_{S(k)}$. If $y \notin S(k)$ there exists a $k$-simplex $\Delta$ such that $y \in$ intv $\Delta \subset S$. Consider the convex cone $C=\{\beta y+(\lambda-\beta+1) z: \beta, \lambda \geqq 0\}$, which has vertex $z$ and is contained in the subspace $L^{\prime}$ with basis $\{p, y\}$. Since $S \cap$ intv $p y^{\infty}=$ $\varnothing, \Delta$ must intersect $L^{\prime}$ in some line other than $L(p, y)$; thus, $S \cap$ intv $C \neq \varnothing$. There exists a linear functional $f$ defined on $L_{k+1}$ such that $f(q)=1$ for every $q \in L(p, y)$; clearly $0 \notin L(p, y)$ since $p y \not \subset S$ and $0 \in$ $c k S$. The continuous linear functional $f_{1}$, the restriction of $f$ to $L^{\prime}$, attains a maximum on the compact set $C \cap S$ at some point $w \in$ intv $C$. Let $H=[f: f(w)]$. Since $H \cap C \cap S$ is a compact subset of the 1-dimensional set $H \cap L^{\prime}$, there exists a minimal closed line segment in intv $C$ which contains $H \cap C \cap S$. Each endpoint of this segment, which may be degenerate, must be a point in $S(k)$. Let $v$ be one of these endpoints. The points $p, y, z$ and $v$ are in $L^{\prime}$. If $p v \subset S$, then the fact that $0 \in c k S$ implies that $z \in \operatorname{conv}\{0, p, v\} \subset S$, a contradiction. Hence, $p v \not \subset S$ and $p \notin S_{S(k)}$. Therefore, $S \backslash c k S \subset S \backslash S_{S(k)}$, which gives the desired equality, since clearly $c k S \subset S_{S(k)}$.

It is not always sufficient to consider only the set of familiar extreme points $S(1)$ as a star-generating set for $c k S$. For example, in $E_{3}$ let $S$ be the union of three closed faces of a 3 -simplex. In some cases, proper subsets of $S(k)$ exist which will star-generate $c k s$. However, characterizing such subsets may be very difficult, as indicated by the following example.

EXAmple 1. In the plane $E_{2}$ let $B_{u}$ be the upper closed unit half-disc, $B_{r}$ the right closed unit half-disc. Let

$$
\begin{aligned}
T_{1} & =\operatorname{conv}\left[\left\{-2 e_{1}\right\} \cup\left(B_{r}+\left(2 e_{1}+e_{2}\right)\right)\right], \\
T_{2} & =\operatorname{conv}\left[\left\{-2 e_{2}\right\} \cup\left(B_{u}+\left(2 e_{2}-e_{1}\right)\right)\right], \\
S & =T_{1} \cup T_{2} \cup\left(-T_{1}\right) \cup\left(-T_{2}\right) .
\end{aligned}
$$

Then any star-generating subset of $S(1)$ must contain four distinct 
sequences of carefully chosen extreme points.

THEOREM 2. If $S$ is a compact star-shaped set in $L_{n}$, and dim $(S) \geqq 3$, then $S(n-1)$ is an uncountable set.

Proof. Without loss of generality, it can be assumed that $0 \in$ $c k S$. Since $\operatorname{dim}(S) \geqq 3$ there exists some point $x \in S, x \neq 0$. If $\beta x \in S(n-1)$ for every $\beta \in(0,1)$, then $S(n-1)$ is uncountable. Otherwise, consider some $w=\beta x$ such that $w \notin S(n-1)$. Then there exists an $(n-1)$-simplex $\Delta$ such that $w \in \operatorname{intv} \Delta \subset S$. Since $n-1 \geqq 2$ there exists a nondegenerate line segment $z w \subset \Delta$ such that $z w \cap 0 x=$ $\{w\}$. There exists a linear functional $f$ on $L_{n}$ such that

$$
f(w)=f(z)=1 .
$$

There exists a point $y \in[f: 0]$ such that the set $\{y, z, w\}$ is linearly independent. For each $\lambda \in[0,1]$ consider the subspace $L(\lambda)$ of $L_{n}$ with basis $\{y, \lambda z+(1-\lambda) w\}$. Let $f_{\lambda}$ be the restriction of $f$ to $L(\lambda)$. The set $L(\lambda) \cap S$ is compact; hence; $f_{\lambda}$ attains a maximum on $L(\lambda) \cap S$ at some point $u, f_{\lambda}(u) \geqq 1$. Since $\operatorname{dim}(L(\lambda) \cap[f: f(u)])=1$ and

$$
L(\lambda) \cap S \cap[f: f(u)]
$$

is compact, there exists a minimal closed line segment in $L(\lambda)$ which contains $L(\lambda) \cap[f: f(u)] \cap S$. This line segment must have at least one endpoint, which must belong to $S(n-1)$. For each pair of distinct real numbers $\lambda, \mu$ in $[0,1], L(\lambda) \cap L(\mu) \subset[f ; 0]$. There exists points $p_{\lambda} \in L(\lambda) \cap S(n-1), p_{\mu} \in L(\mu) \cap S(n-1)$ such that $f\left(p_{\lambda}\right) \geqq 1, f\left(p_{\mu}\right) \geqq 1$, which implies that $p_{\lambda} \neq p_{\mu}$. Thus, the set $S(n-1)$ is uncountable.

Theorem 3. Let $S$ be a closed subset of a linear topological space $L$ and let $T$ be a subset of $S$ that star-generates ckS, which may be empty. If $M$ is a dense subset of $T$, then $M$ star-generates $c k S$.

Proof. Since $M \subset T$ then clearly $S_{T} \subset S_{M}$. Suppose that $M$ is a proper subset of $T$ and $c k S$ is a proper subset of $S_{M}$. Then there exists a point $q \in S_{M} \backslash S_{T}$. But $S_{T}=S_{M} \cap S_{T \backslash M}$; thus $q \notin S_{T \backslash M}$. This implies that $q \notin S_{x}$ for some $x \in T \backslash M$. Since $q \in S_{M}, M \subset S_{q}$, which is closed. Hence, $x \in T \subset \operatorname{cl} M \subset S_{q}$, which implies that $x q \subset S$ and that $q \in S_{x}$, a contradiction. Therefore, $c k S=S_{M}$.

THEOREM 4. If $S$ is a compact star-shaped subset of a normed linear space $L$, then any subset $T$ of $S$ which star-generates the convex kernel of $S$ contains a countable subset $M$ which also star-generates the convex kernel of $S$. 
Proof. The norm of $L$ induces a metric on $L$. The compact set $S$ can be considered as a compact metric space, where space is now used in the topological sense. The compact metric space is separable, which implies that $S$ is second countable [2]. Any nonempty subset $T$ of $S$ is a second countable topological space with the relative topology, which implies that $T$ is separable. There exists a countable subset $M$ of $T$ such that $T \subset \mathrm{cl} M$. Theorem 3 implies that $M$ stargenerates $c k S$ and the theorem is proved.

CoRollary. Let $S$ be a compact star-shaped subset of $L_{k+1}$. Then there exists a countable subset of $S(k)$ which star-generates ckS.

Klee [3] introduced the concept of relative extreme point.

Definition 3. If $S$ and $T$ are subsets of a linear space $L$, then $x \in S$ is said to be extreme in $S$ relative to $T$ if there do not exist points $y \in S, z \in T$ such that $x \in \operatorname{intv} y z$.

If $S$ is a star-shaped set, exk $S$ will denote the points of $S$ which are extreme relative to $c k S$, and $E_{S}=(\operatorname{exk} S) \backslash c k S$.

THEOREM 5. Let $S$ be a compact nonconvex star-shaped set in a locally convex space $L$. Then $C=S$, where

$$
C=\bigcup_{y \in E_{S}} \operatorname{conv}(c k S \cup\{y\}) \text {. }
$$

Proof. Since $E_{S} \subset S$, conv $(\operatorname{cks} S \cup\{y\}) \subset S$ for each $y \in E_{S}$. Thus, $C \subset S$. Consider $z \in c k S \cup \operatorname{exk} S$; since $E_{S} \neq \varnothing$, as shown below, $z \in$ $C$. Let $K=c k S$. Suppose that $z \in S \backslash(c k S \cup \operatorname{exk} S)$ and without loss of generality, suppose that $z=0$. Since $K$ is compact and convex, $K^{*}$ and $-K^{*}$ are closed convex cones with vertex 0 , where $K^{*}=$ $\{\lambda x: x \in K, \lambda \geqq 0\}$. Since $z \notin \operatorname{exk} S$ there exist points $x \in K$ and $w \in S$ such that $0 \in \operatorname{intv} x w$. Clearly $w \in-K^{*} \backslash\{0\}, S \cap\left(-K^{*} \backslash\{0\}\right) \neq \varnothing$ and $S \cap\left(-K^{*}\right)$ is compact. Let $u$ be an arbitrary point in $-K^{*} \backslash\{0\}$; since $L$ is locally convex and $K^{*}$ is closed and convex, there exists a closed hyperplane $H=[f: f(u)]$ such that $u \in H$ and $H \cap K^{*}=\varnothing$, where $f$ is a continuous linear functional. It can be assumed that $f\left(K^{*}\right) \leqq 0$, which implies that $f(u)>0$. The functional $f$ then attains a maximum on $S \cap\left(-K^{*}\right)$ at some point $v \in S \cap\left(-K^{*}\right)$. Suppose that $v \notin \operatorname{exk} S$. There exist points $p \in K, q \in S$ such that $v \in \operatorname{intv} p q$. Since $v \in-K^{*}, v=-\lambda p^{\prime}, p^{\prime} \in K, \lambda>0$, and

$$
v=\alpha p+(1-\alpha) q, \quad 0<\alpha<1 .
$$

Therefore, $v=-\lambda p^{\prime}=\alpha p+(1-\alpha) q$ and $q=\tau q^{\prime}$, where $\tau<0$ and $q^{\prime} \in K$. Thus, $q \in S \cap\left(-K^{*}\right)$. But it can be easily shown that 
$f(q)>f(v)$, which contradicts the fact that $f(v) \geqq f(x)$ for each $x \in$ $S \cap\left(-K^{*}\right)$. Hence, $v \in(\operatorname{exk} S) \cap\left(-K^{*}\right)$ and $0 \in C$, which implies that $S \subset C$. This inclusion, along with the one given earlier, implies that $S=C$.

The following result shows that the set $E_{S}$ is minimal in its use in Theorem 5 .

THEOREM 6. Let $S$ be a compact nonconvex star-shaped set in a locally convex space $L$. If $T$ is a proper subset of $E_{S}$ then

$$
C(T)=\bigcup_{y \in T} \operatorname{conv}(c k S \cup\{y\})
$$

is a proper subset of $S$.

Proof. Consider any proper subset $T$ of $E_{S}$; there exists some point $x \in E_{S} \backslash T$. If $x \in C(T)$ there exists some $y \in T$ such that $x \in$ conv $(c k S \cup\{y\})$. Hence, $x=\lambda z+(1-\lambda) y$, where $\lambda \in[0,1], z \in c k S$. But $\lambda \in(0,1)$ since $x \notin c k S \cup T$. This implies that $x \notin \operatorname{exk} S$, a contradiction. Thus, $x \notin C(T)$, which must be a proper subset of $S$.

\section{BIBLIOGRAPHY}

1. E. Asplund, $A$ k-extreme point is the limit of k-exposed points, Israel J. Math. 1 (1963), 161-162.

2. D. W. Hall and G. L. Spencer II, Elementary topology, John Wiley and Sons, Inc., New York, 1955.

3. V. L. Klee, Relative extreme points, Proceedings of the International Symposium on Linear Spaces, Pergamon Press, New York, 1961.

4. F. A. Valentine, Convex sets, McGraw-Hill, New York, 1964.

Received May 13, 1968. This work was done to partially fulfill the requirements for the degree of Doctor of Philosophy at Oklahoma State University under the direction of Professor E. K. McLachlan. During this time the author was an NSF Cooperative Graduate Fellow.

OKLahoma State University

Texas Technological College 



\section{PACIFIC JOURNAL OF MATHEMATICS}

\section{EDITORS}

H. ROYDEN
Stanford University
Stanford, California

\section{R. R. PHELPS}

University of Washington

Seattle, Washington 98105
J. DUGUNDJI

Department of Mathematics

University of Southern California

Los Angeles, California 90007

\section{RICHARD ARENS}

University of California

Los Angeles, California 90024

\section{ASSOCIATE EDITORS}

E. F. BeCKenbaCh
B. H. NEUMANN

F. WOLF
K. YoshidA

\section{SUPPORTING INSTITUTIONS}

\author{
UNIVERSITY OF BRITISH COLUMBIA \\ CALIFORNIA INSTITUTE OF TECHNOLOGY \\ UNIVERSITY OF CALIFORNIA \\ MONTANA STATE UNIVERSITY \\ UNIVERSITY OF NEVADA \\ NEW MEXICO STATE UNIVERSITY \\ OREGON STATE UNIVERSITY \\ UNIVERSITY OF OREGON \\ OSAKA UNIVERSITY \\ UNIVERSITY OF SOUTHERN CALIFORNIA
}

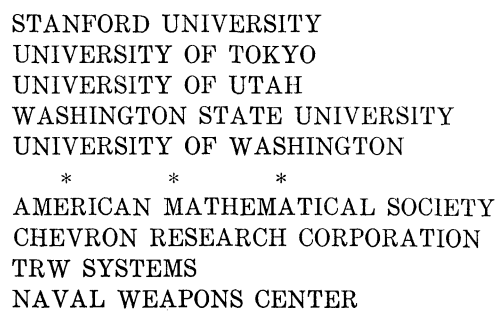

The Supporting Institutions listed above contribute to the cost of publication of this Journal, but they are not owners or publishers and have no responsibility for its content or policies.

Mathematical papers intended for publication in the Pacific Journal of Mathematics should be in typed form or offset-reproduced, double spaced with large margins. Underline Greek letters in red, German in green, and script in blue. The first paragraph or two must be capable of being used separately as a synopsis of the entire paper. It should not contain references to the bibliography. Manuscripts, in duplicate if possible, may be sent to any one of the four editors. Please classify according to the scheme of Math. Rev. 36, 1539-1546. All other communications to the editors should be addressed to the managing editor, Richard Arens, University of California, Los Angeles, California, 90024.

50 reprints are provided free for each article; additional copies may be obtained at cost in multiples of 50 .

The Pacific Journal of Mathematics is published monthly. Effective with Volume 16 the price per volume (3 numbers) is $\$ 8.00$; single issues, $\$ 3.00$. Special price for current issues to individual faculty members of supporting institutions and to individual members of the American Mathematical Society: $\$ 4.00$ per volume; single issues $\$ 1.50$. Back numbers are available.

Subscriptions, orders for back numbers, and changes of address should be sent to Pacific Journal of Mathematics, 103 Highland Boulevard, Berkeley, California, 94708.

PUBLISHED BY PACIFIC JOURNAL OF MATHEMATICS, A NON-PROFIT CORPORATION

Printed at Kokusai Bunken Insatsusha (International Academic Printing Co., Ltd.), 7-17, Fujimi 2-chome, Chiyoda-ku, Tokyo, Japan. 


\section{Pacific Journal of Mathematics \\ Vol. 29, No. $2 \quad$ June, 1969}

Bruce Langworthy Chalmers, On boundary behavior of the Bergman kernel function and related domain functionals ................... 243

William Eugene Coppage, Peirce decomposition in simple Lie-admissible power-associative rings .............................. 251

Edwin Duda, Compactness of mappings...................... 259

Earl F. Ecklund Jr., On prime divisors of the binomial coefficient......... 267

Don E. Edmondson, A modular topological lattice ............... 271

Phillip Alan Griffith, A note on a theorem of Hill ................... 279

Marcel Herzog, On finite groups with independent cyclic Sylow

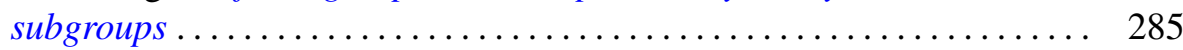

James A. Huckaba, Extensions of pseudo-valuations................. 295

S. A. Huq, Semivarieties and subfunctors of the identity functor ........ 303

I. Martin (Irving) Isaacs and Donald Steven Passman, Finite groups with small character degrees and large prime divisors. II ............ 311

Carl Kallina, A Green's function approach to perturbations of periodic

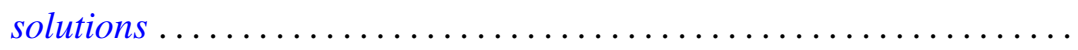

$\mathrm{Al}$ (Allen Frederick) Kelley, Jr., Analytic two-dimensional subcenter manifolds for systems with an integral ....................

Alistair H. Lachlan, Initial segments of one-one degrees ............ 351

Marion-Josephine Lim, Rank k Grassmann products ............. 367

Raymond J. McGivney and William Henry Ruckle, Multiplier algebras of

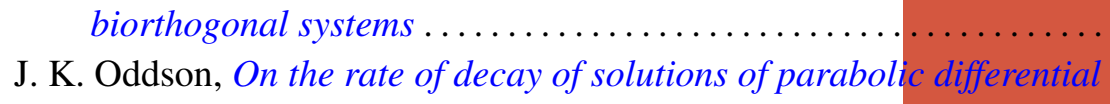

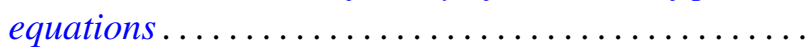

Helmut R. Salzmann, Geometries on surfaces ........... .

Annemarie Schlette, Artinian, almost abelian groups and their groups of automorphisms ............................

Edgar Lee Stout, Additional results on modules over polydisc algebras ...

Lajos Tamássy, A characteristic property of the sphere . .

Mark Lawrence Teply, Some aspects of Goldie's torsion theory. ...

Freddie Eugene Tidmore, Extremal structure of star-shaped sets ...

461

Leon Jarome Weill, Unconditional and shrinking bases in locally convex spaces... 